Analyse \& Kritik 02/2011 (c) Lucius \& Lucius, Stuttgart) S. 473-476

Julian Culp

\title{
Comment on Lukas Meyer and Pranay Sanklecha. Individual Expectations and Climate Justice*
}

Meyer and Sanklecha's elaborate article $^{1}$ addresses an issue of practical importance for all of us who are living in highly industrialized countries, and who are formulating or revising our life plans and long-term projects. It examines whether the expectation of people living in highly industrialized countries to be able to continue to emit greenhouse gases at their current average level in the future (Expectation E) is epistemically and politically legitimate, and morally permissible. Such an investigation is directly relevant for any formulation or revision of a life plan or long-term project, since any such plan or project should take into consideration whether it is accessible both from an empirical and from a normative point of view. Thus, as many potential life plans and long-term projects are based upon Expectation E, people living in highly industrialized countries must determine whether Expectation $\mathrm{E}$ is realistically achievable and consistent with relevant normative standards. For if Expectation E turned out to be either epistemically illegitimate, politically illegitimate, or morally impermissible, then — certainly prima facie - people living in highly industrialized countries would have good reason to formulate and revise their life plans and long-term projects in such a way that these would avoid being based upon Expectation E. Therein lies the crucial practical import of Meyer and Sanklecha's article.

This comment engages with two aspects of Meyer and Sanklecha's relatively brief discussion of the political legitimacy of Expectation E and leaves aside for the most part their analyses of the epistemic legitimacy and moral permissibility of Expectation E. First, it suggests framing the discussion of the political legitimacy of Expectation E differently than Meyer and Sanklecha, i.e. not in terms of its legal permissibility given the assumptions that highly industrialized states or

\footnotetext{
* I owe many thanks to Lukas Meyer and Pranay Sanklecha as well as to Robert Jubb for very helpful comments on a penultimate version of this comment.

1 The page numbers in brackets refer to Meyer and Sanklecha's article in this issue. The abbreviations 'Expectation E', 'level X', 'Phi' and 'Shi' are adopted from their article, but are nevertheless introduced in this comment in due course.
} 
regions ('Shi') are legitimate authorities that enforce legitimate system of laws. And then, second, it draws attention to a potential tension among some of the assumptions that Meyer and Sanklecha make in the course of their discussion of the political legitimacy of Expectation E.

To begin, recall that Meyer and Sanklecha start from the conceptual point that political legitimacy is "the question of justified state coercion" and mainly understand this question "in the case of Expectation $\mathrm{E}$ as being a question of legal permissibility given the background assumptions that the legal system, and the authority which establishes and maintains it, are both themselves legitimate" (456). The question of the political legitimacy of Expectation E, that is, Meyer and Sanklecha understand as the question whether the people living in highly industrialized countries or regions ('Phi') can lawfully act on Expectation E within the legal systems of Shi.

Now, if the question about the political legitimacy of Expectation E amounts to the question whether Shi permits Phi acting on Expectation E, then it appears to become unclear what the normative question about the political legitimacy of Expectation E is. After all, according to this understanding of the political legitimacy of Expectation E, the political legitimacy of Expectation E hinges upon whether laws in Shi permit or forbid acting on Expectation E. This raises the question whether there is an alternative approach to the question of the political legitimacy of Expectation E, which would be capable of examining Expectation E from a more distinctively normative point of view. I think that there is and will propose it in a second.

But before that, consider that Meyer and Sanklecha could point out that they also pursue the further question "what are the conditions that need to be fulfilled for Shi to be in a position to justifiably coerce Phi into not fulfilling Expectation E?" (455); and they could argue that this question is a genuinely normative one. This does not seem to be the case, however, because the assumptions that Shi constitute legitimate authorities that enforce legitimate laws (cf. 455-6), already seem to render the answer to this question positivist: it says that states can justifiably coerce Phi into not fulfilling Expectation E by introducing laws that prohibit Phi from doing so. So this way of framing the question of the political legitimacy of Expectation E also results in the question whether Shi enact laws that permit Phi to act on Expectation E.

How, then, could we formulate a more normative question about the justifiability of state coercion in regard to Expectation E? I suggest that the question "when is that power [i.e. state coercion] appropriately exercised?" (Rawls 2005, 137) could in this context mean considering whether justifiable state coercion is consistent with Phi's acting on Expectation E. In other words, we could also ask this question: Can we conceive of Shi as legitimate political units, that is, as states that justifiably coerce their members, and at the same time assume that these members act on Expectation E? Different from Meyer and Sanklecha's question, this question would not assume the legitimacy of the authority and the laws of Shi. Rather, it would ask whether acting on Expectation E is consistent with Shi's legitimacy and would examine whether Shi must prohibit the 
fulfillment of Expectation E in order to render its coercive power "appropriately exercised". ${ }^{2}$

An analogy between the political legitimacy of Expectation E and the political legitimacy of private financing of political campaigns might help to bring out the appeal of formulating the question of justifiable state coercion in the way that the last paragraph suggested. If one asks whether the private financing of political campaigns is politically legitimate, then one arguably wants to know whether this practice is consistent with justifiable state coercion. That is, arguably, one wants to examine whether, on the assumption that private financing of political campaigns remains in place, coercive political power could be appropriately exercised. While such an examination, of course, would also have to rely on a host of empirical considerations, the result would be a response to the genuinely normative question as to whether justifiable state coercion requires the prohibition of private financing of political campaigns. Analogously, examining the political legitimacy of Expectation E by asking whether "appropriately exercised" state coercion would have to prohibit Phi's acting on Expectation E also addresses a distinctively normative question.

Note that Meyer and Sanklecha have implicitly answered this question negatively. For by assuming that the authorities and laws of Shi are legitimate, whether or not they institute laws that hinder the fulfillment of Expectation E, they grant that acting on Expectation $\mathrm{E}$ is consistent with the appropriate exercise of coercive power. But, or so it seems, they do not set out the arguments for the claim that political units that do not enact such laws may nevertheless be considered legitimate authorities making legitimate laws. A more normatively oriented discussion of the political legitimacy of Expectation E, I think, would be well advised to carry out the examination suggested above and test whether Phi's acting on Expectation E renders the political units of Shi illegitimate or not.

Finally, let me point at a potential tension that grows out of the assumption that Shi are legitimate authorities and another three assumptions that Meyer and Sanklecha make when discussing the political legitimacy of Expectation E.

One of the other assumptions is that the current average per capita level of emissions in Shi (level X), which Phi expect to be able to continue to emit in the future, is above the just per capita level of emissions (cf. 451). A second one is that "the legitimate system of laws established [...] by Shi allows Phi to act on Expectation E" (456). And a further assumption, which appears only towards the end of the article, says that "the satisfaction of "some substantive conditions of justice"" (468) is part of any set of criteria of legitimacy.

These four assumptions could come into conflict with each other. This is because by acknowledging that level $\mathrm{X}$ is above the just per capita level of emission, Shi's legally permitting Phi to act on Expectation E could constitute a substantive injustice, more specifically, an intergenerational one. And this, contrary to the first of the four assumptions stated above, could render Shi illegitimate

2 There is a further way of understanding the political legitimacy of Expectation E, which asks whether it is legitimate for Shi to prohibit Phi acting on Expectation E, rather than whether it is illegitimate for Shi not to prohibit Phi acting on Expectation E. 
authorities, to the extent that the fulfillment of 'some substantive conditions of justice' conditions the legitimacy of political units like Shi, as put forward in the fourth assumption. Meyer and Sanklecha themselves make clear that "[p]eople whenever they live have basic rights that may not be violated" (451). So Shi's failure to act upon the duties that correlate with these basic rights could manifest a serious neglect of substantive "reasons of intergenerational justice" (451), and hence could undermine Shi's presupposed legitimacy.

However, this conflict would only arise if one considered the violation of these demands of intergenerational injustice as an impediment to complying with 'some substantive conditions of justice'. Thus, whether there actually is a tension among these four assumptions depends upon how grave one judges the violation of standards of intergenerational justice to be. That is, Meyer and Sanklecha's assumptions do not necessarily conflict each other, but could, if one were to assess Shi's infringements of the basic rights of future people as so serious that these infringements alone would undermine the legitimacy of Shi. It would be illuminating to learn from Meyer and Sanklecha which reasons apparently led them to thinking that such infringements do not necessarily erode Shi's legitimacy. They would thereby further clarify their already very instructive normative framework for considering our expectations about our ability to emit greenhouse gases in the future.

\section{Bibliography}

Rawls, J. (2005[1993]), Political Liberalism, New York 